\title{
Robust linear PID controller for satellite attitude stabilisation and attitude tracking control
}

\author{
Research Center of Satellite Technology, \\ Harbin Institute of Technology, \\ Harbin, 150001, China \\ Email: liyouhahaha@163.com \\ Email: sunzhaowei@hit.edu.cn \\ Email: yed@hit.edu.cn \\ *Corresponding author
}

You Li, Zhaowei Sun and Dong Ye*

\begin{abstract}
In this paper, a linear robust PID controller is presented for satellite attitude stabilisation and attitude tracking control. The PID controller presented in this paper has the advantage that: 1) the Lyapunov function is modified thus the stability of the system is easier to prove; 2) the constraints of the parameters are broadened thus parameters are easier to choose comparing with existing methods; 3 ) the controller is robust to bounded disturbance torque and the satellite inertia matrix; 4) the controller is linear thus it is convenient for practical application. The proof of the stability and the constraints of the parameters are given in this paper. The simulation results verify the feasibility of the controller presented in this paper.
\end{abstract}

Keywords: PID controller; robust linear controller; attitude stabilisation control; attitude tracking control.

Reference to this paper should be made as follows: Li, Y., Sun, Z. and Ye, D. (2016) 'Robust linear PID controller for satellite attitude stabilisation and attitude tracking control', Int. J. Space Science and Engineering, Vol. 4, No. 1, pp.64-75.

Biographical notes: You $\mathrm{Li}$ is a $\mathrm{PhD}$ candidate at Harbin Institute of Technology. His research is mainly about satellite attitude control and attitude determination algorithms for fast manoeuvre satellites.

Zhaowei Sun is a Professor at Harbin Institute of Technology. His research is mainly about dynamic modelling and control for complex spacecraft.

Dong Ye is an Assistant Professor at Harbin Institute of Technology. His research is mainly about attitude control algorithms for flexible spacecraft.

\section{Introduction}

PD control algorithm is the most mature and widely used control algorithm in satellite attitude control system. For satellite attitude stabilisation and tracking control, PD control with negative feedback is global asymptotically stable. In order to improve the accuracy 
of the system, the integration item is brought into the control law. However, the appearance of the integration item makes it difficult to prove the stability of the system.

Jin and Sun (2009) presented a PID controller for flexible spacecraft attitude tracking in 2009. In his work, a Lyapunov function with coupled item of angular velocity, quaternion and integration item was presented and based on this Lyapunov function the integration item was eliminated in the derivative of Lyapunov function. Thus, the order of the system was reduced and the stability of the system was proved. In his work in 2008 (Jin et al., 2008), he presented a PID controller for decentralised satellite formation control. In this work, the Lyapunov function with coupled item was also used to achieve the goal of reducing the system order. In Kamesh (2004), the author constructed a modified Lyapunov function with integration item and simplified the proof of the system stability. However, this work was mainly focused on rigid spacecraft. In Clarke et al. (1997), the PID controller for attitude tracking issue was discussed. The attitude tracking system was treated as non-autonomous system and its strict stability proof was given. In this work, the stability of discontinuous system was discussed and the stability of control law with sign function was proved. In Vadali and Junkins (1990) and Chaturvedi et al. (2006), the non-autonomous system stability was discussed and the Lyapunov function was constructed in a relatively complicated way to prove the convergence of the system state. It is worth noticing that most of these PID controllers presented were nonlinear. In Li et al. (2014), a parameter optimisation method based on genetic algorithm for PID controller is presented for attitude control systems. In Su et al. (2011), a nonlinear PID controller robust to disturbance torque was presented for quadrotor aircraft and the strict stability proof was given. However, in this work, the accurate inertia matrix was needed to design the controller. In Sakamoto et al. (2006) and Zhang et al. (2010), the fuzzy PID controller and robust PID controller is presented for helicopter attitude control. The author considered the complicated environment and modified traditional PID control algorithm in order to enhance its robustness. In Lu et al. (2008), the author designed PID controller based on the idea of sliding mode for rigid satellite.

In this paper, the attitude stabilisation and attitude tracking issue is discussed for rigid satellite. Inspired by Jin and Sun (2009) and Jin et al. (2008), the Lyapunov function with coupled items is constructed and modified. Thus the proof of stability and the constraints of parameters become easier to achieve. Also, in order to hold the advantage of robustness to inertia matrix of PD controller, the controller designed in this paper does not need the accurate value of satellite inertia matrix. The simulation results examine the feasibility of the algorithm.

\section{Dynamic and kinetic model}

The dynamic model of satellite could be written as follow:

$$
J \dot{\omega}+\omega^{\times} J \omega=u+d
$$

where $J$ is the inertia matrix of satellite which is a symmetric matrix, $d$ is the unknown disturbance torque with upper bound $|d|_{i}<\bar{d}$. The product matrix $r^{\times}$of vector $r$ is defined as 


$$
r^{\times}=\left[\begin{array}{ccc}
0 & -r_{3} & r_{2} \\
r_{3} & 0 & -r_{1} \\
-r_{2} & r_{1} & 0
\end{array}\right]
$$

The product matrix has an important property which will be used in the later part of this paper that the eigenvalues of $r^{\times}$satisfies

$$
\begin{aligned}
& \lambda\left(r^{\times}\right)=0, \pm\|r\| i \\
& \left|\lambda\left(r^{\times}\right)\right|_{\max }=\|r\|
\end{aligned}
$$

The kinetic model of satellite could be written as follow:

$$
\dot{q}=\left[\begin{array}{c}
\dot{q}_{0} \\
\dot{q}_{v}
\end{array}\right]=\left[\begin{array}{c}
-\frac{1}{2} q_{v}^{T} \omega \\
\frac{1}{2}\left(q_{0} I_{3}+q_{v}^{\times}\right) \omega
\end{array}\right]=\frac{1}{2}\left[\begin{array}{c}
-q_{v}^{T} \\
q_{0} I_{3}+q_{v}^{\times}
\end{array}\right] \omega
$$

In satellite attitude tracking issue, the error quaternion and error angular velocity are defined as follow:

$$
\begin{aligned}
& q_{e}=q_{d}^{*} \otimes q=\left[\begin{array}{c}
q_{d 0} q_{0}+q_{d v}^{T} q_{v} \\
q_{d 0} q_{v}-q_{0} q_{d v}-q_{d v} \times q_{v}
\end{array}\right] \\
& R\left(q_{e}\right)=\left(q_{e 0}^{2}-q_{e v}^{T} q_{e v}\right) I_{3}+2 q_{e v} q_{e v}^{T}-2 q_{e v} q_{e v}^{\times} \\
& \omega_{e}=\omega-R\left(q_{e}\right) \omega_{d}
\end{aligned}
$$

where $q_{d}$ and $\omega_{d}$ are desired quaternion and angular velocity.

The model of error angular velocity and quaternion could be written as follow:

$$
\begin{aligned}
& J \dot{\omega}_{e}+J R\left(q_{e}\right) \dot{\omega}_{d}-J \omega_{e}^{\times} R\left(q_{e}\right) \omega_{d}+\left[\omega_{e}+R\left(q_{e}\right) \omega_{d}\right]^{\times} J\left[\omega_{e}+R\left(q_{e}\right) \omega_{d}\right]=u+d \\
& \dot{q}_{e}=\left[\begin{array}{c}
\dot{q}_{e 0} \\
\dot{q}_{e v}
\end{array}\right]=\left[\begin{array}{c}
-\frac{1}{2} q_{e v}^{T} \omega_{e} \\
\frac{1}{2}\left(q_{e 0} I_{3}+q_{e v}^{\times}\right) \omega_{e}
\end{array}\right]=\frac{1}{2}\left[\begin{array}{c}
-q_{e v}^{T} \\
q_{e 0} I_{3}+q_{e v}^{\times}
\end{array}\right] \omega_{e}
\end{aligned}
$$

\section{PID attitude stabilisation control law}

The linear PID attitude stabilisation control law presented as follow:

$$
u=-k_{d} \omega-k_{p} q_{v}-k_{I} \vartheta-\bar{d} \operatorname{sgn}\left(\beta \omega+l_{2} q_{v}\right)
$$

where $k_{d}, k_{p}, k_{l}, \beta, l_{2}$ are all positive scalars and variable $\vartheta$ is defined as follow:

$$
\dot{\vartheta}=c_{1} \omega+c_{2} q_{v}-\frac{1}{2} \alpha F \omega
$$


where $c_{1}, c_{2}, \alpha$ are positive scalars and $F$ is defined as follow:

$$
F=q_{0} I_{3}+q_{v}^{\times}
$$

The eigenvalues of satisfies following equation:

$$
\begin{aligned}
& \lambda(F)=q_{0}, q_{0} \pm i \sqrt{1-q_{0}^{2}} \\
& |\lambda(F)|_{\max }=1
\end{aligned}
$$

where $\lambda(A)$ represents the eigenvalues of matrix $A$.

Consider equation (8), the control torque $u$ could be rewritten as follow:

$$
\begin{aligned}
u & =-k_{d} \omega=k_{p} q_{v}=k_{I} \int\left(c_{1} \omega+c_{2} q_{v}\right) d t+\alpha k_{I} \int \dot{q}_{v} d t-\bar{d} \operatorname{sgn}\left(\beta \omega+l_{2} q_{v}\right) \\
& =-k_{d} \omega-k_{p} q_{v}-k_{I} \int\left(c_{1} \omega+c_{2} q_{v}\right) d t+\alpha k_{I} \int\left(q_{v}-q_{v}(0)\right)-\bar{d} \operatorname{sgn}\left(\beta \omega+l_{2} q_{v}\right)
\end{aligned}
$$

Based on equation (12), it can be found that the control law presented in this paper is a linear PID control law without nonlinear item.

The next step is to prove that by choosing proper parameters, system (1), (4), (8) is global asymptotically stable.

Choose Lyapunov function as follow:

$$
V=2 l_{1}\left(1-q_{0}\right)+\frac{1}{2} \beta \omega^{T} J \omega+l_{2} q_{v}^{T} J \omega+l_{3}\left(\vartheta+\alpha q_{v}\right)^{T}\left(\vartheta+\alpha q_{v}\right)
$$

where $l_{1}, l_{2}, l_{3}, \alpha, \beta$ are positive scalar.

Considering that

$$
1-q_{0} \geq \frac{1}{2}\left(1-q_{0}^{2}\right), \quad \forall q_{0} \in[-1,1]
$$

It can be got that

$$
\begin{aligned}
V & \geq l_{1} q_{v}^{T} q_{v}+\frac{1}{2} \beta \omega^{T} J \omega+l_{2} q_{v}^{T} J \omega+l_{3}\left(\vartheta+\alpha q_{v}\right)^{T}\left(\vartheta+\alpha q_{v}\right) \\
& \geq l_{1} q_{v}^{T} q_{v}+\frac{1}{2} \beta \omega^{T} J \omega+l_{2} q_{v}^{T} J \omega \\
& =\left[\begin{array}{ll}
\omega^{T} & q_{v}^{T}
\end{array}\right]\left[\begin{array}{cc}
\frac{1}{2} \beta \lambda_{\min }(J) I_{3} & \frac{1}{2} l_{2} \lambda_{\max }(J) I_{3} \\
\frac{1}{2} l_{2} \lambda_{\max }(J) I_{3} & l_{1} I_{3}
\end{array}\right]\left[\begin{array}{l}
\omega \\
q_{v}
\end{array}\right]
\end{aligned}
$$

Thus, if following inequality is satisfied, $V$ is positive definite.

$$
\frac{1}{2} \beta l_{1} \lambda_{\min }(J)-\frac{1}{4} l_{2}^{2} \lambda_{\max }^{2}(J)>0
$$

Calculate the derivative of $V$, it can be got that 


$$
\begin{aligned}
\dot{V} & =l_{1} \omega^{T} q_{v}+\beta \omega^{T}\left(-\omega^{\times} J \omega+u+d\right)+\frac{1}{2} l_{2} \omega^{T} J F \omega+l_{2} q_{v}^{T}\left(-\omega^{\times} J \omega+u+d\right) \\
& +2 l_{3}\left(\vartheta+\alpha q_{v}\right)^{T}\left(c_{1} \omega+c_{2} q_{v}-\frac{1}{2} \alpha F \omega+\frac{1}{2} \alpha F \omega\right) \\
& =\left(\beta \omega^{T}+l_{2} q_{v}^{T}\right)\left(-k_{d} \omega-k_{p} q_{v}-k_{I} \vartheta\right)+\left(\beta \omega^{T}+l_{2} q_{v}^{T}\right)\left(-\bar{d} \operatorname{sgn}\left(\beta \omega+l_{2} q_{v}\right)+d\right) \\
& +\frac{1}{2} l_{2} \omega^{T} J F \omega-l_{2} q_{v}^{T} \omega^{\times} J \omega+2 l_{3}\left(\vartheta+\alpha q_{v}\right)^{T}\left(c_{1} \omega+c_{2} q_{v}\right)+l_{1} \omega^{T} q_{v} \\
& \leq-\omega^{T}\left(\beta k_{d} I_{3}-\frac{1}{2 l_{2} J F}\right) \omega-q_{v}^{T}\left(l_{2} k_{p} I_{3}-2 \alpha c_{2} l_{2} l_{3}\right) q_{v}+\left(2 c_{1} l_{3}-\beta k_{O}\right) \vartheta^{T} \omega \\
& +\left(2 c_{2} l_{3}-l_{2} k_{I}\right) \vartheta^{T} q_{v}+\left(l_{1}-\beta k_{p}-l_{2} k_{d}+2 \alpha c_{1} l_{3}\right) q_{v}^{T} \omega-l_{2} q_{v}^{T} \omega^{\times} J \omega
\end{aligned}
$$

Choose proper parameters to satisfy following equation.

$$
2 l_{3} c_{1}-\beta k_{I}=2 l_{3} c_{2}-l_{2} k_{I}=l_{1}-\beta k_{p}-l_{2} k_{d}+2 \alpha c_{1} l_{3}=0
$$

Assume that the norm of angular velocity satisfies $\|\omega\| \leq \bar{\omega}$, and consider property (3) and (10), equation (17) could be transformed to

$$
\dot{V} \leq-\left[\begin{array}{ll}
\omega^{T} & q_{v}^{T}
\end{array}\right]\left[\begin{array}{cc}
\beta k_{d} I_{3}-\frac{1}{2} l_{2} \lambda_{\max }(J) I_{3} & \frac{1}{2} l_{2} \bar{\omega} \lambda_{\max }(J) I_{3} \\
\frac{1}{2} l_{2} \bar{\omega} \lambda_{\max }(J) I_{3} & l_{2}\left(k_{p}-\alpha k_{I}\right) I_{3}
\end{array}\right]\left[\begin{array}{l}
\omega \\
q_{v}
\end{array}\right]
$$

Thus, if the following inequality is satisfied, $\dot{V}$ is negative definite.

$$
l_{2}\left(\beta k_{d}-\frac{1}{2} l_{2} \lambda_{\max }(J)\right)\left(k_{p}-\alpha k_{I}\right)-\frac{1}{4} l_{2}^{2} \bar{\omega}^{2} \lambda_{\max }^{2}(J)>0
$$

Thus, if (16), (18) and (20) are satisfied, $V>0, \dot{V}<0$, the system is global asymptotically stable.

\section{PID attitude tracking control law}

In this paper, the tracking target is cooperative which means $q_{d}$ and $\omega_{d}$ are all known. Assume the norm of real angular velocity is upper bounded which means $\|\omega\| \leq \bar{\omega}$.

The PID attitude tracking law could be written as follow:

$$
\begin{aligned}
u= & -k_{d} \omega_{e}-k_{p} q_{e v}-k_{I} \vartheta_{e}-\bar{d} \operatorname{sgn}\left(s_{e}\right)-\lambda_{\max }(J) s_{e} \odot(R \dot{\omega} d) \\
& -\lambda_{\max }(J) s_{e} \odot\left(\omega_{e}^{\times} R \omega_{d}\right)-\bar{\omega} \lambda_{\max }(J) s_{e} \odot \omega_{e}
\end{aligned}
$$

where $\vartheta_{e}$ and $s_{e}$ satisfies

$$
\begin{aligned}
& \dot{\vartheta}_{e}=c_{1} \omega_{e}+c_{2} q_{e v}-\frac{1}{2} \alpha F_{e} \omega_{e} \\
& F_{e}=q_{e 0} I_{3}+q_{e v}^{\times} \\
& s_{e}=\beta \omega_{e}+l_{2} q_{e v}
\end{aligned}
$$


And $\lambda_{\max }(J)$ in equation (21) represents the maximum eigenvalue of $J$. The operator $\odot$ is defined as follow:

$$
a \odot b=\left[\begin{array}{lll}
\operatorname{sgn}\left(a_{1} b_{1}\right) b_{1} & \operatorname{sgn}\left(a_{2} b_{2}\right) b_{2} & \operatorname{sgn}\left(a_{3} b_{3}\right) b_{3}
\end{array}\right]^{T}
$$

The next step is to prove the stability of control law (21). Choose Lyapunov function as follow:

$$
V_{e}=2 l_{1}\left(1-q_{e 0}\right)+\frac{1}{1} \beta \omega_{e}^{T} J \omega_{e}+l_{2} q_{e v}^{T} J \omega_{e}+l_{3}\left(\vartheta_{e}+\alpha q_{e v}\right)^{T}\left(\vartheta_{e}+\alpha q_{e v}\right)
$$

Consider equation (14), it can be got that

$$
\begin{aligned}
V_{e} & \geq l_{1} q_{e v}^{T} q_{e v}+\frac{1}{2} \beta \omega_{e}^{T} J \omega_{e}+l_{2} q_{e v}^{T} J \omega_{e}+l_{3}\left(\vartheta_{e}+\alpha q_{e v}\right)^{T}\left(\vartheta_{e}+\alpha q_{e v}\right) \\
& \geq l_{1} q_{e v}^{T} q_{e v}+\frac{1}{2} \beta \omega_{e}^{T} J \omega_{e}+l_{2} q_{e v}^{T} J \omega_{e} \\
& =\left[\begin{array}{ll}
\omega_{e}^{T} & q_{e v}^{T}
\end{array}\right]\left[\begin{array}{cc}
\frac{1}{2} \beta \lambda_{\min }(J) I_{3} & \frac{1}{2} l_{2} \lambda_{\max }(J) I_{3} \\
\frac{1}{2} l_{2} \lambda_{\max }(J) I_{3} & l_{1} I_{3}
\end{array}\right]\left[\begin{array}{l}
\omega_{e} \\
q_{e v}
\end{array}\right]
\end{aligned}
$$

Thus, if following inequality is satisfied, $V_{e}$ is positive definite.

$$
\frac{1}{2} \beta l_{1} \lambda_{\min }(J)-\frac{1}{4} l_{2}^{2} \lambda_{\max }^{2}(J)>0
$$

Calculate the derivative of $V_{e}$, it can be got that

$$
\begin{aligned}
\dot{V}_{e}= & -2 l_{1} \dot{q}_{e 0}+\beta \omega_{e}^{T} J \dot{\omega}_{e}+l_{2} \omega_{e}^{T} J \dot{q}_{e v}+l_{2} q_{e v}^{T} J \dot{\omega}_{e}+2 l_{3}\left(\vartheta_{e}+\alpha q_{e v}\right)^{T}\left(\dot{\vartheta}_{e}+\alpha \dot{q}_{e v}\right) \\
= & l_{1} q_{e v}^{T} \omega_{e}+l_{2} \omega_{e}^{T} J F_{e} \omega_{e}+\left(\beta \omega_{e}+l_{2} q_{e v}\right)^{T} J \dot{\omega}_{e} \\
& +2 l_{3}\left(\vartheta_{e}+\alpha q_{e v}\right)^{T}\left(c_{1} \omega_{e}+c_{2} q_{e v}-\frac{1}{2} \alpha F_{e} \omega_{e}+\frac{1}{2} \alpha F_{e} \omega_{e}\right) \\
& =l_{1} q_{e v}^{T} \omega_{e}+\frac{1}{2} l_{2} \omega_{e}^{T} J F_{e} \omega_{e}+2 l_{3} c_{1} \vartheta_{e}^{T} \omega_{e}+2 l_{3} c_{2} \vartheta_{e}^{T} q_{e v}+2 l_{3} c_{1} \alpha q_{e v}^{T} \omega_{e}+2 l_{3} c_{2} \alpha q_{e v}^{T} q_{e v} \\
& +\left(\beta \omega_{e}+l_{2} q_{e v}\right)^{T}\left(u+d-J R \dot{\omega}_{d}+J \omega_{e}^{\times} R \omega_{d}-\omega^{\times} J \omega\right) \\
& =-\omega_{e}^{T}\left(k_{d} \beta I_{3}-\frac{1}{2} l_{2} J F_{e}\right) \omega_{e}-q_{e v}^{T}\left(l_{2} k_{p}-2 l_{3} c_{2} \alpha\right) q_{e v}+\left(2 c_{1} l_{3}-\beta k_{I}\right) \vartheta_{e}^{T} \omega_{e} \\
& +\left(2 c_{2} l_{3}-l_{2} k_{I}\right) \vartheta_{e}^{T} q_{e v}+q_{e v}^{T}\left(l_{1}-\beta k_{p}-l_{2} k_{d}+2 \alpha c_{1} l_{3}\right) \omega_{e} \\
& +\left(\beta \omega_{e}+l_{2} q_{e v}\right)^{T}\left(\tau+d-J R \dot{\omega}_{d}+J \omega_{e}^{\times} R \omega_{d}-\omega^{\times} J \omega\right)
\end{aligned}
$$

Choose proper parameters to satisfy following equation

$$
2 l_{3} c_{1}-\beta k_{I}=2 l_{3} c_{2}-l_{2} k_{I}=l_{1}-\beta k_{p}-l_{2} k_{d}+2 \alpha c_{1} l_{3}=0
$$

Equation (27) could be transformed to 


$$
\begin{aligned}
\dot{V}_{e}= & -\omega_{e}^{T}\left(k_{d} \beta I_{3}-\frac{1}{2} l_{2} J F_{e}\right) \omega_{e}-q_{e v}^{T}\left(l_{2} k_{p}-l_{2} k_{I} \alpha\right) q_{e v} \\
& +s_{e}^{T}\left(\tau+d-J R \dot{\omega}_{d}+J \omega_{e}^{\times} R \omega_{d}-\omega^{\times} J \omega\right)
\end{aligned}
$$

where $\tau$ satisfies

$$
\tau=-\bar{d} \operatorname{sgn}\left(s_{e}\right)-\lambda_{\max }(J) s_{e} \odot\left(R \dot{\omega}_{d}\right)-\lambda_{\max }(J) s_{e} \odot\left(\omega_{e}^{\times} R \omega_{d}\right)-\bar{\omega} \lambda_{\max }(J) s_{e} \odot \omega_{e}
$$

Considering the following property

$$
\begin{aligned}
-s_{e}^{T}\left(s_{e} \odot r\right) \pm s_{e}^{T} r & =-s_{e}^{T}\left[\operatorname{sgn}\left(s_{e 1} r_{1}\right) r_{1} \quad \operatorname{sgn}\left(s_{e 2} r_{2}\right) r_{2} \quad \operatorname{sgn}\left(s_{e 3} r_{3}\right) r_{3}\right]^{T} \pm s_{e}^{T} r \\
& =-\sum s_{e i} r_{i} \operatorname{sgn}\left(s_{e i} r_{1}\right) \pm \sum s_{e i} r_{i} \\
& =-\sum\left|s_{e i} r_{i}\right| \pm \sum s_{e i} r_{i} \leq 0
\end{aligned}
$$

It can be got that

$$
\begin{aligned}
& -\bar{d}_{e}^{T} \operatorname{sgn}\left(s_{e}\right)+s_{e}^{T} d \leq 0 \\
& -\lambda_{\max }(J) s_{e}^{T} s_{e} \odot\left(R \dot{\omega}_{d}\right)-s_{e}^{T} J R \dot{\omega}_{d} \leq-s_{e}^{T} s_{e} \odot\left(J R \dot{\omega}_{d}\right)-s_{e}^{T} J R \dot{\omega}_{d} \leq 0 \\
& -\lambda_{\max }(J) s_{e}^{T} s_{e} \odot\left(\omega_{e}^{\times} R \omega_{d}\right)+s_{e}^{T} J \omega_{e}^{\times} R \omega_{d} \leq-s_{e}^{T} s_{e} \odot\left(J \omega_{e}^{\times} R \omega_{d}\right)+s_{e}^{T} J \omega_{e}^{\times} R \omega_{d} \leq 0 \\
& -\bar{\omega} \lambda_{\max }(J) s_{e}^{T} s_{e} \odot \omega_{e}-s_{e}^{T} \omega^{\times} J \omega \leq-s_{e}^{T} s_{e} \odot\left(\omega^{\times} J \omega\right)-s_{e}^{T} \omega^{\times} J \omega \leq 0
\end{aligned}
$$

Thus, equation (29) could be transformed to

$$
\begin{aligned}
\dot{V}_{e} & \leq-\omega_{e}^{T}\left(k_{d} \beta I_{3}-\frac{1}{2} l_{2} J F_{e}\right) \omega_{e}-q_{e v}^{T}\left(l_{2} k_{p}-l_{2} k_{I} \alpha\right) q_{e v} \\
& \leq-\omega_{e}^{T}\left(k_{d} \beta-\frac{1}{2} l_{2} \lambda_{\max }(J)\right) \omega_{e}-q_{e v}^{T}\left(l_{2} k_{p}-l_{2} k_{I} \alpha\right) q_{e v}
\end{aligned}
$$

Thus, if following inequality is satisfied, $\dot{V}_{e}<0$.

$$
k_{d} \beta-\frac{1}{2} l_{2} \lambda_{\max }(J)>0, k_{p}-\alpha k_{I}>0
$$

Therefore, the attitude tracking system is global asymptotically stable.

Comparing the necessary parameter constraints in attitude stabilisation and attitude tracking issues, it can be found that the constraints of $V$ positive definite and the constraints to eliminate integration items are all the same. The difference is that the constraint of $\dot{V}$ negative definite. Based on equation (15) and (34), it can be found that the constraint in attitude tracking issue is easier to satisfy.

\section{Simulation}

Set the system parameters for attitude stabilisation control as follow. 


$$
\begin{aligned}
& J=\operatorname{diag}\left(\left[\begin{array}{lll}
50 & 75 & 100
\end{array}\right]\right), \bar{\omega}=0.2, T=500, t_{-} \text {sample }=0.5 \\
& \alpha=1, \beta=0.05, k_{d}=20, k_{p}=5, k_{I}=1, \bar{d}=0.001 \\
& c_{1}=0.25, c_{2}=0.05, l_{1}=0.4, l_{2}=0.01, l_{3}=0.1 \\
& \omega_{\text {initial }}=\left[\begin{array}{lllll}
0.1 & -0.05 & -0.04
\end{array}\right]^{T}, q_{\text {initial }}=\left[\begin{array}{llll}
0.51 & 0.3 & 0.4 & -0.7
\end{array}\right]^{T}
\end{aligned}
$$

The simulation of attitude stabilisation control law is given from Figure 1 to Figure 3.

Based on Figure 1 to Figure 3, it can be found that the angular velocity and attitude quaternion converges to zero. Except the initial ten control cycle, the norm of control torque is constrained in an acceptable range.

Then, set the system parameters for attitude tracking control as follow:

$$
\begin{aligned}
& J=\operatorname{diag}\left(\left[\begin{array}{lll}
50 & 75 & 100
\end{array}\right]\right), \bar{\omega}=0.2, T=500, t_{-} \text {sample }=0.5 \\
& \alpha=1, \beta=0.05 ., k_{d}=20, k_{p} 5, k_{I}=1, \bar{d}=0.001 \\
& c_{1}=0.25, c_{2}=0.05, l_{1}=0.4, l_{2}=0.01, l_{3}=0.1 \\
& \omega_{\text {initial }}=\left[\begin{array}{llll}
0.05 & 0.05 & -0.1
\end{array}\right]^{T}, q_{\text {initial }}=\left[\begin{array}{llll}
0.5 & -0.5 & \sqrt{3} / 3 & \sqrt{6} / 6
\end{array}\right]^{T} \\
& \omega_{d}=\left[\begin{array}{lllll}
0.01 & -0.005 & 0
\end{array}\right]^{T}, \dot{\omega}_{d}=0_{3 \times 1}, q_{d}=\left[\begin{array}{llll}
1 & 0 & 0 & 0
\end{array}\right]^{T}
\end{aligned}
$$

The simulation of attitude tracking control is given from Figure 4 to Figure 6.

Figure 1 Curve of angular velocity (see online version for colours)

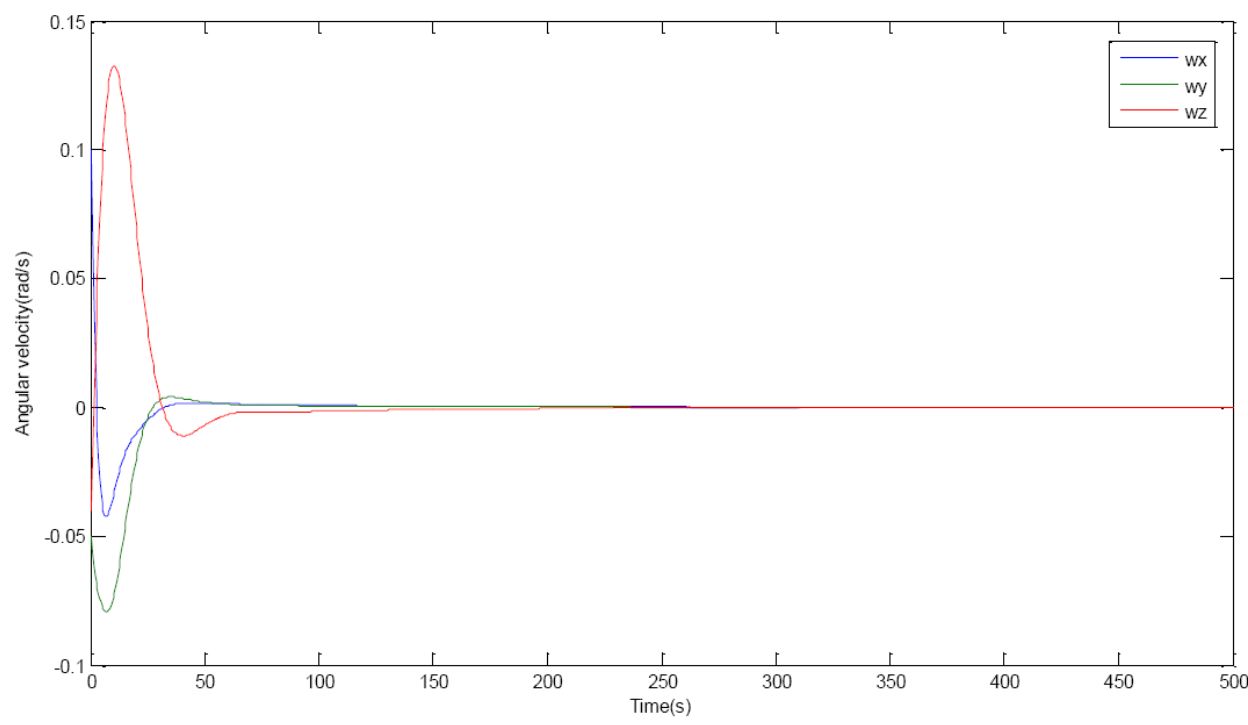


Figure 2 Curve of quaternion (see online version for colours)

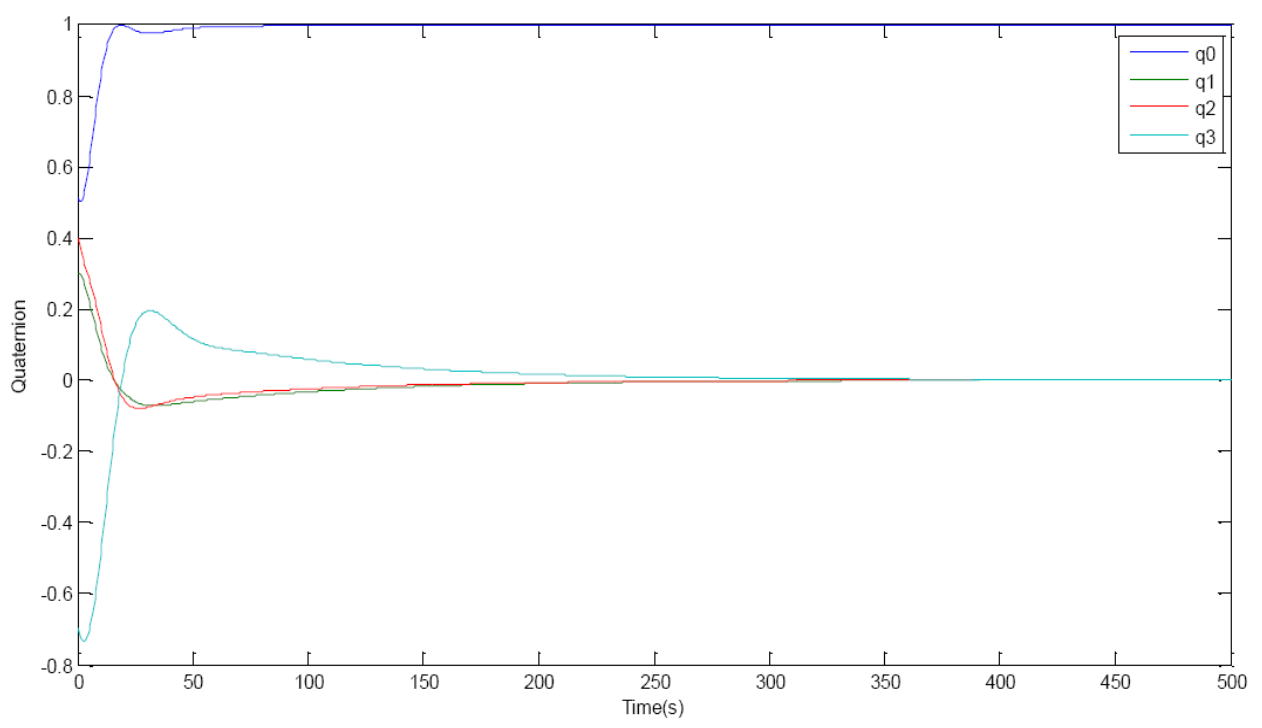

Figure 3 Curve of control torque (see online version for colours)

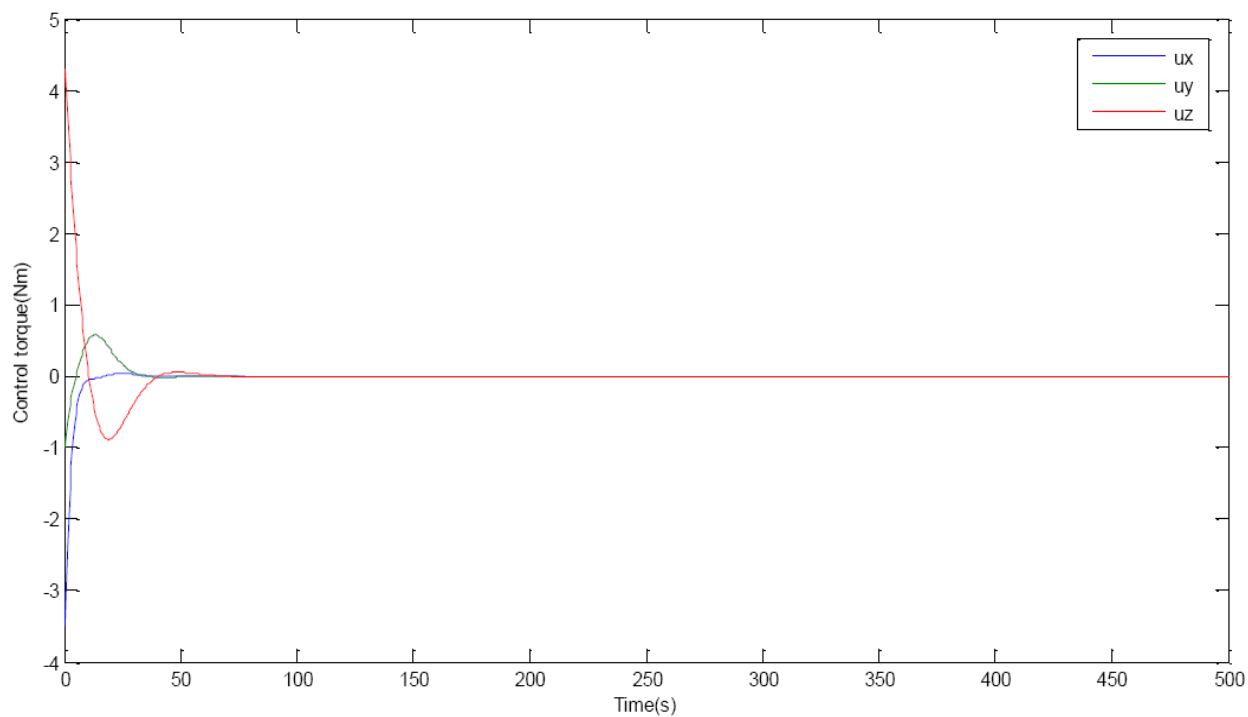

Based on Figure 4 to Figure 6, it can be found that the error angular velocity and error quaternion converges to zero. Except the initial 20 control cycles, the norm of control torque is constrained in an acceptable range. 
Figure 4 Curve of error angular velocity (see online version for colours)

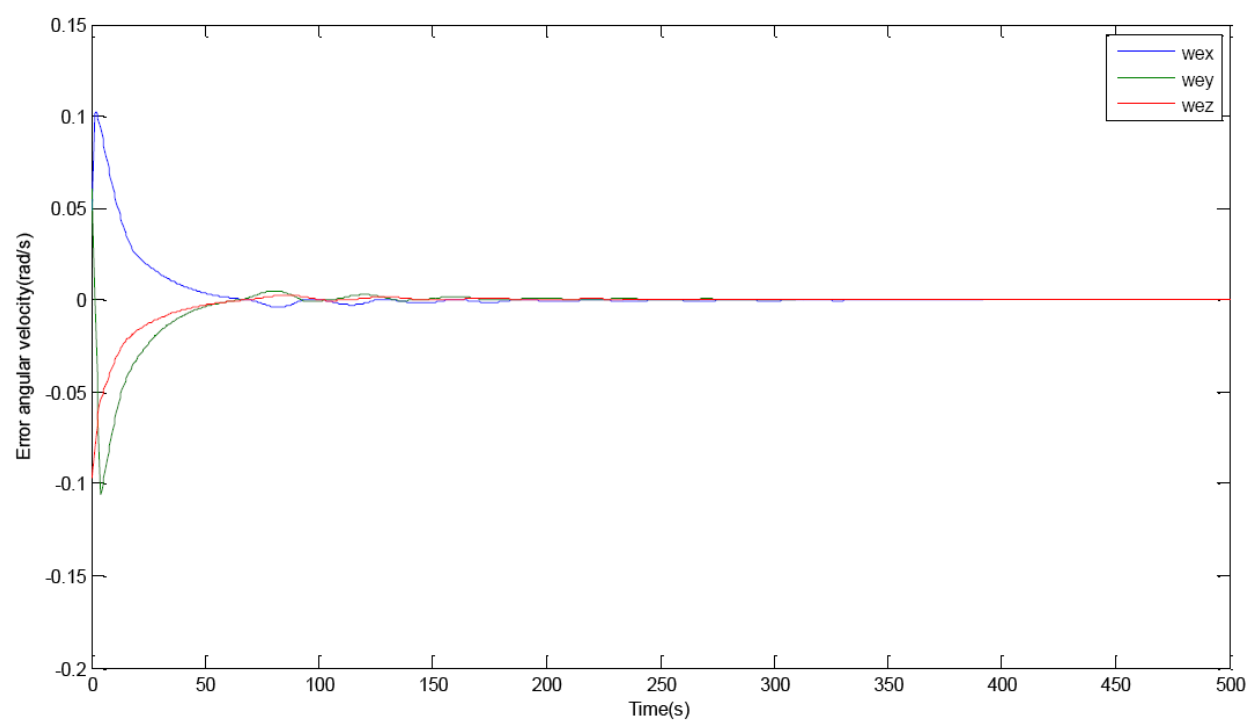

Figure 5 Curve of error quaternion (see online version for colours)

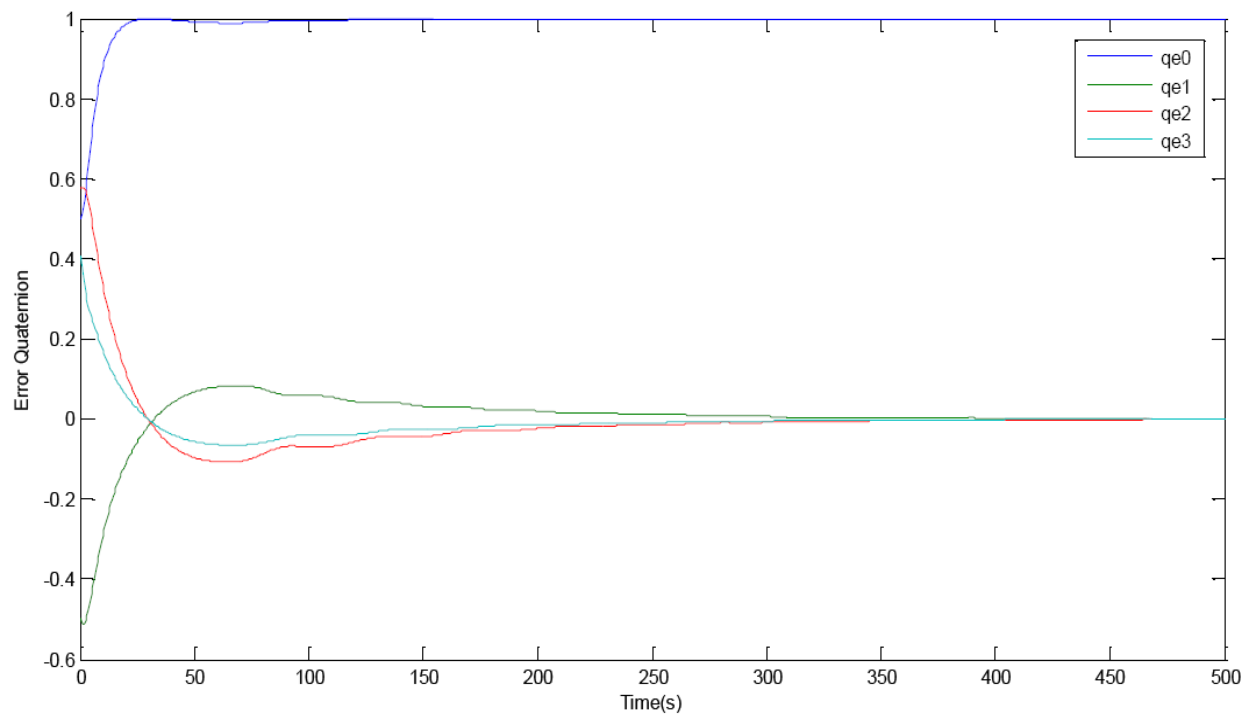


Figure 6 Curve of control torque (see online version for colours)

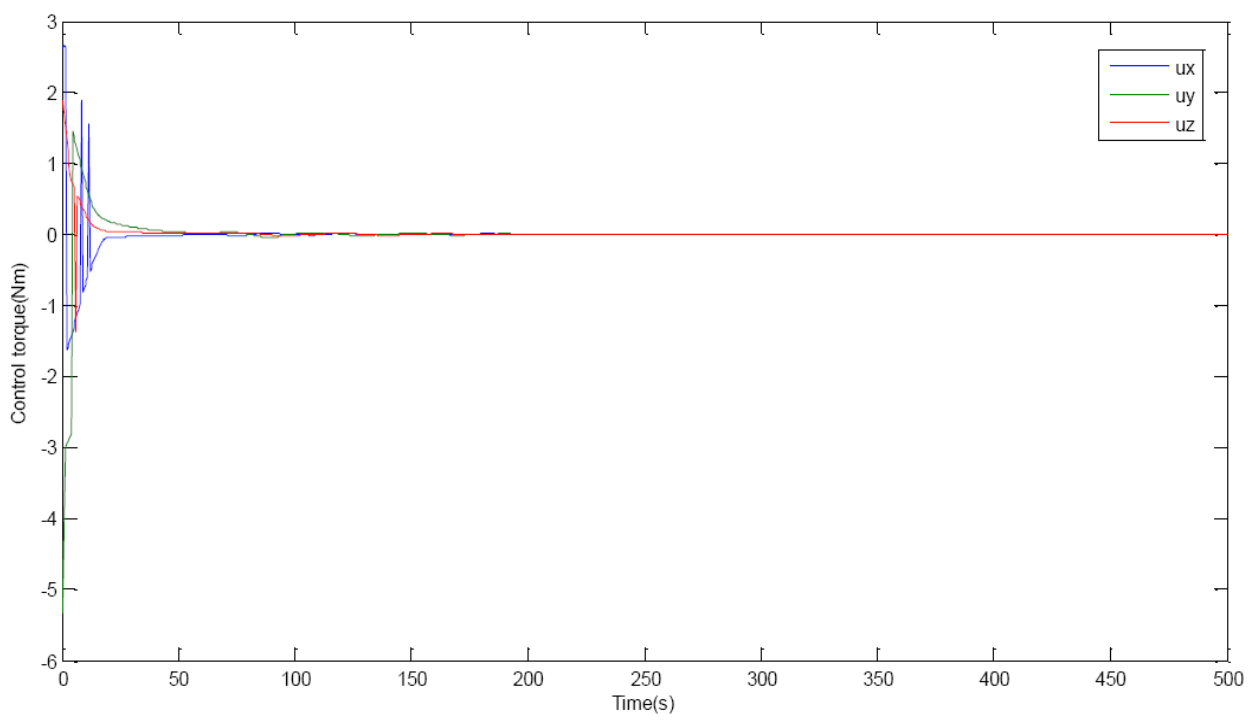

\section{Conclusions}

In this paper, a method for design PID attitude stabilisation and attitude tracking control law is given. The traditional Lyapunov function is modified thus the constraints on control parameters are easier to satisfy comparing with existing methods (Jin and Sun, 2009; Jin et al., 2008). Also, the integration item in control law is modified to eliminate the integration items in Lyapunov function. Both of these two improvements make it easier to prove the stability and choose proper parameters.

The stability proof of the control law is given in this paper. Also, the constraints of the system parameters are given in the proof of the system stability. The simulation results indicate that when choosing proper parameters the system is asymptotically stable.

The attitude stabilisation and attitude tracking PID control law in this paper has the advantages that:

1 easier to prove the stability

2 easier to choose the control parameters

3 only the maximum eigenvalue of inertia matrix is needed which means the controller is robust to the inertia matrix uncertainty

4 the controller is linear thus it is convenient for practical application. 


\section{References}

Chaturvedi, N.A., Sanyal, A.K., Chellappa, M., Valk, J.L. and McClamroch, N.H. (2006) 'Adaptive tracking of angular velocity for a planar rigid body with unknown models for inertia and input nonlinearity', IEEE Transactions on Control Systems Technology, Vol. 14, No. 4, pp.613-627.

Clarke, F.H., Ledyaev, Y.S., Sontag, E.D. and Subbotin, A.I. (1997) 'Asymptotic controllability implies feedback stabilization', IEEE Transactions on Automatic Control, Vol. 42, No. 10, pp.1394-1407.

Jin, E. and Sun, Z. (2009) 'Robust attitude tracking control of flexible spacecraft for achieving globally asymptotic stability', International Journal of Robust and Nonlinear Control, Vol. 19, No. 11, pp.1201-1223.

Jin, E., Jiang, X. and Sun, Z. (2008) 'Robust decentralized attitude coordination control of spacecraft formation', Systems \& Control Letters, Vol. 57, No. 7, pp.567-577.

Kamesh, S. (2004) 'Nonlinear PID-like control for spacecraft attitude regulation', The Journal of Astronautical Sciences, Vol. 52, No. 2, pp.61-74.

Li, X., Qu, S. and Cai, L. (2014) 'Studying of PID parameter optimization method for attitude controller of quadrotor', Journal of Computational Information Systems, Vol. 10, No. 18, pp.7805-7812.

Lu, J., Li, C. and Ma, G. (2008) 'Attitude sliding mode PID controller design for satellite regulation', Journal of Harbin Institute of Technology, Vol. 40, No. 7, pp.1009-1012.

Sakamoto, T., Katayama, H. and Ichikawa, A. (2006) 'Attitude control of a helicopter model by robust PID controllers', Proceedings of the 2006 IEEE International Symposium on Intelligent Control.

Su, J., Fan, P. and Cai, K. (2011) 'Attitude control of quadrotor aircraft via nonlinear PID', Journal of Beijing University of Aeronautics and Astronautics, Vol. 37, No. 9, pp.1054-1058.

Vadali, S.R. and Junkins, J.L. (1990) 'Closed loop slewing of flexible spacecraft', Journal of Guidance, Control, and Dynamics, Vol. 13, No. 1, pp.57-65.

Zhang, L., Bi, S. and Yang, H. (2010) 'Fuzzy-PID control algorithm of the helicopter model flight attitude control', 2010 Chinese Control and Decisions Conference. 